Gamma-ray Emission After Neutron Capture: Modeling for Excitation Energies Involving Strongly Overlapping Resonances

J. E. Escher

September 15, 2013 
This document was prepared as an account of work sponsored by an agency of the United States government. Neither the United States government nor Lawrence Livermore National Security, LLC, nor any of their employees makes any warranty, expressed or implied, or assumes any legal liability or responsibility for the accuracy, completeness, or usefulness of any information, apparatus, product, or process disclosed, or represents that its use would not infringe privately owned rights. Reference herein to any specific commercial product, process, or service by trade name, trademark, manufacturer, or otherwise does not necessarily constitute or imply its endorsement, recommendation, or favoring by the United States government or Lawrence Livermore National Security, LLC. The views and opinions of authors expressed herein do not necessarily state or reflect those of the United States government or Lawrence Livermore National Security, LLC, and shall not be used for advertising or product endorsement purposes.

This work performed under the auspices of the U.S. Department of Energy by Lawrence Livermore National Laboratory under Contract DE-AC52-07NA27344. 


\title{
Gamma-ray emission after neutron capture: Modeling for excitation energies involving strongly overlapping resonances
}

\author{
Jutta E. Escher \\ Lawrence Livermore National Laboratory, Livermore, CA 94550
}

(Dated: September 19, 2013)

\begin{abstract}
Current libraries of neutron-capture gamma-ray spectra contain little or no data for incident neutron energies above the thermal region. An effort is described for modeling gamma-ray spectra following neutron capture to the energy regime of strongly overlapping resonances in the compound nucleus.
\end{abstract}

\section{MOTIVATION}

Gamma-ray emission spectra following the interaction of neutrons with an unknown sample of material give valuable information on the composition of the material, provided reliable and complete libraries of gamma-ray spectra for neutron capture on a wide range of isotopes are available.

Within the framework of an ongoing effort [4, 14] to update and complete present libraries with the latest experimental gamma-ray information, complemented by a modeling effort, an area in clear need of improvement was identified. Specifically, it was recognized that current libraries of neutron-capture gamma-ray spectra contain little, no, or unreliable data for neutrons incident with energies larger than thermal energy $\left(E_{n}=0.0253 \mathrm{eV}\right.$ for $\left.\mathrm{T}=293^{\circ} \mathrm{K}\right)$.

\section{COMPOUND-NUCLEAR DECAY BY GAMMA EMISSION}

A compound nucleus $A+1$, created by neutron capture on a target $A$, decays by gamma-ray emission, neutron evaporation, and possibly other channels, such as charged-particle emission or fission. Gamma-ray emission often dominates at low-neutron energies, with competition by neutron evaporation becoming stronger with increasing excitation energy.

Thermal neutron capture results in gamma-ray spectra that are frequently measured and recorded in libraries for retrieval and use in transport codes. The measurements are also used to determine thermal capture cross sections. Limitations in experimental set-ups and analyses require that the spectra are complemented by calculations which model the gamma cascades in the decaying nuclei. For thermal capture, this can be done with the code DicEBox [1]. In this approach, nuclear gamma-cascades are simulated by using the Monte-Carlo method. The gamma-decay of an isolated, highly-excited initial level in a medium or heavy nucleus is repeatedly generated for individual, artificial events, and appropriate averages are carried out, with account being taken of the Porter-Thomas fluctuations involved. For capture to the energy regime of resolved resonances in the intermediate compound nucleus, an extension of this approach is underway [16].

At higher energies, the resonances overlap, until a regime is reached where the statistical Hauser-Feshbach approach [5] becomes applicable. The energies at which the transitions from resolved to unresolved resonance region, and from unresolved to Hauser-Feshbach region occur, depends on factors such as the mass of the nucleus and its location relative to closed nuclear shells. This is illustrated in Figure 1, where with increasing neutron energy, regions of resolved resonances, unresolved resonances, and finally Hauser-Feshbach regime are traversed. The latter is indicated by a curve that represents an average.

A variety of codes are available to describe reactions proceeding through this Hauser-Feshbach energy regime, including Talys [10], EmpIRe [6], Stapre [17], etc. Recommended input parameters for model calculations can be found in the literature [2].

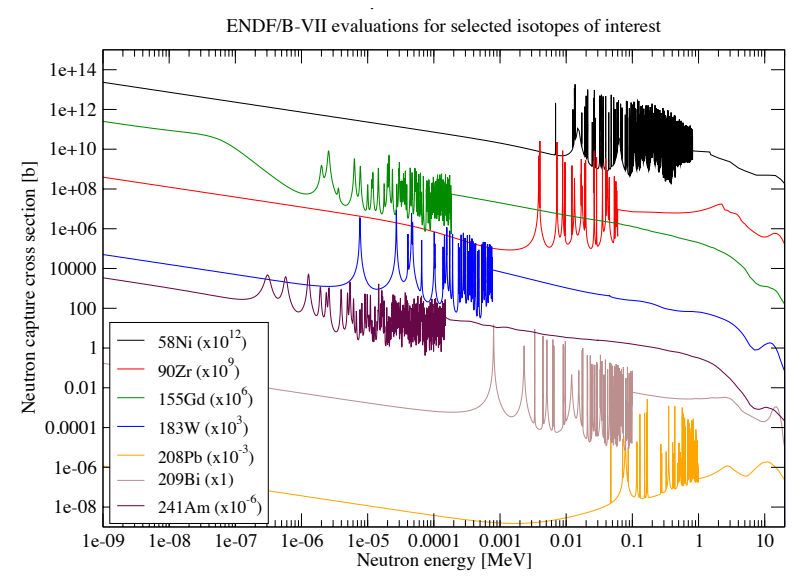

FIG. 1: Typical capture cross sections, for selected isotopes. Shown are evaluated cross sections for neutron energies from thermal to $20 \mathrm{MeV}$. The ENDF/B-VII evaluations, taken from the NNDC database, are calculations based on experimental data.

Modeling the gamma decay of a compound nucleus involves a description of different types of gamma-rays. Figure 2 shows the model space for a typical decay calculation. For thermal capture, primary transitions to discrete levels below $E_{\text {crit }}$, above $E_{\text {crit }}$, and to energy bins above $E_{\text {crit }}$ can occur. Furthermore, transitions be- 
tween energy bins (continuum-to-continuum), as well as transitions from energy bins to discrete levels, can occur. Finally, there are transitions between discrete low-lying states.

Transitions between discrete low-lying states have typically been measured in various experiments. Information on the branchings for these levels is entered into calculations as input. Continuum-to-discrete and continuumto-continuum transitions can be modeled using suitable level density and gamma-ray strength function descriptions. This can be done for both thermal and HauserFeshbach calculations. Primary transitions are sensitive to the detailed structure of the initial, decaying (resonance) state, as well as the final state reached in the transitions. As the initial states are highly-excited manybody states, a reliable theoretical description is not possible. For thermal capture, information on primary transitions can sometimes be obtained from measurements and subsequently incorporated into the modeling effort. For higher neutron energies, this experimental information is typically not available. In the Hauser-Feshbach regime, transitions from the energy bin populated initially by neutron capture to low-lying discrete levels is determined by average quantities, such as level densities and gamma strength functions, and by the quantum numbers of the final states. Consequently, the calculated primary transitions cannot be taken to be precise predictions, but need to be interpreted in a manner that involves averages over a range of neutron energies.

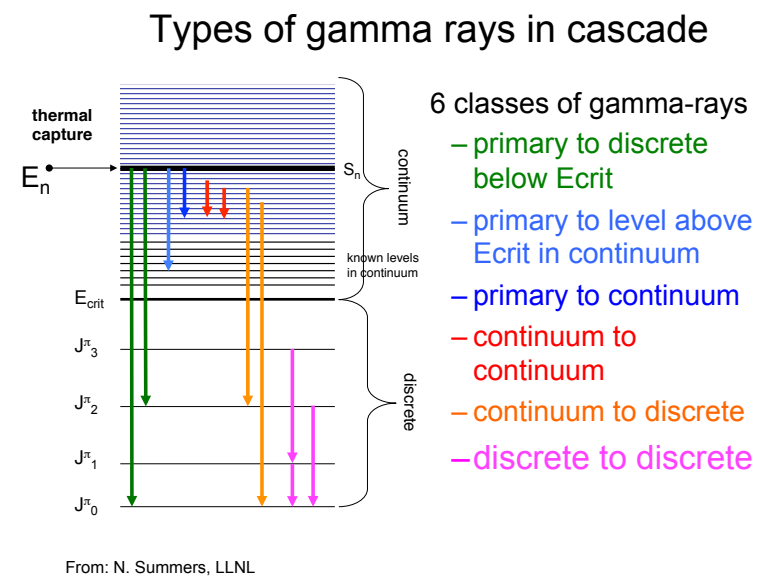

FIG. 2: Gamma-ray spectra for the decay of typical compound nucleus, following thermal neutron capture. Various classes of transitions are shown. $E_{\text {crit }}$ refers to the excitation energy in the compound nucleus below which all discrete levels and their properties (spin, parity, decay branchings) are taken into account. Figure from Ref. [15]

\section{HAUSER-FESHBACH MODELING FOR TUNGSTEN}

Recent capture-gamma ray measurements for Tungsten isotopes are currently being evaluated for thermal neutron capture [7-9]. The modeling described here is designed to complement that effort. Similar calculations have been carried out for Gadolinium, to complement an analogous thermal evaluation [3], but are not shown here.

Hauser-Feshbach calculations were carried out for neutron capture on ${ }^{186} \mathrm{~W}$ with a modified version of the code STAPRE [17]. The code was extended to make it possible to separately calculate primary gamma transitions to discrete levels below $E_{\text {crit }}$, transitions between discrete low-lying states in ${ }^{187} \mathrm{~W}$, and continuum contributions. Primary transitions to discrete levels above $E_{\text {crit }}$ are not predicted by current codes. They are, along with primary transitions to the continuum, contained in the "continuum contributions.' Furthermore, continuum-to-discrete transitions are not expected to show strong structures, so they were not separated out, but included in the 'continuum contributions' as well.

Neutron transmission coefficients, needed to calculate the population of the compound nucleus in the capture reaction as well as for describing the competing neutron evaporation channel, were determined from a coupled-channels calculation using ECIS [13] and the optical model by Koning and Delaroche [11]. Gammaray strength functions and level density parameters were obtained from RIPL-3, the Reference Input Parameter Library for nuclear reaction calculations [2]. Parameters were adjusted to reproduce measured neutron capture cross sections.

Calculations were carried out for neutron energies up to $5 \mathrm{MeV}$. Selected results, for representative neutron energies, are shown in Figure 3. Each panel showns gammaray production cross sections a function of gamma-ray energy. The different panels show the results for different incident neutron energies, for $E_{n}=0.083 \mathrm{MeV}$ (top), 2.195 MeV (middle), and $4.783 \mathrm{MeV}$ (bottom). Primary gamma-ray transitions are shown in maroon, discrete transitions are indicated in green, and the continuum contributions are given in blue.

The overall energy-dependence of the neutron-capture cross section strongly influences the cross sections for the individual gammas. The drop in the gamma spectra with increasing neutron energy is clearly visible. Also clearly shown is the shift in the gamma-ray energies for the primary transitions with increasing energy. Transitions between discrete levels remain fixed in energy, but drop in magnitude. The overall behavior of the cross sections is as expected.

Results for Gadolinium show similar trends. 

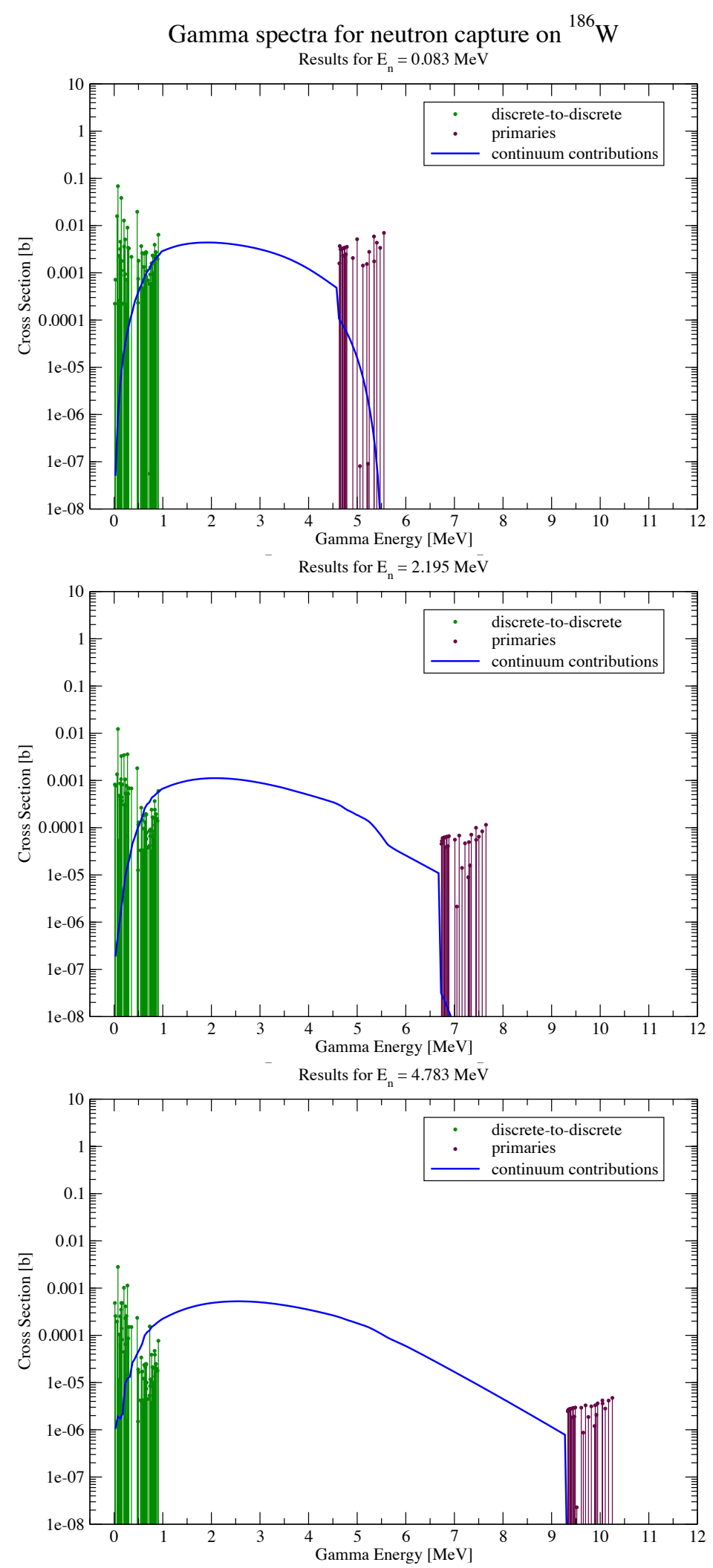

FIG. 3: Gamma-ray spectra for the decay of ${ }^{187} \mathrm{~W}$, following neutron capture at energies $E_{n}=0.083 \mathrm{MeV}$ (top), $2.195 \mathrm{MeV}$ (middle), and $4.783 \mathrm{MeV}$ (bottom). Primary transitions are shown in maroon, transitions between discrete low-lying states in green, and the remaining continuum contributions are indicated in blue. 


\section{OUTLOOK}

This work has focused on gamma-ray modeling for the decay of a compound nucleus excited to an energy regime where resonance strongly overlap and a Hauser-Feshbach description is appropriate. To achieve consistency with the modeling at thermal energies and in the regime of isolated resonances, use is being made of gamma-ray branching ratios that have been verified in the modeling of the thermal capture process. Similarly, it is important to select level densities and gamma-ray strength functions that reproduce measured low-energy average resonance parameters, such as $D_{0}$, the s-wave resonance spacing, and $\Gamma_{\gamma}$, the average radiative width [12]. To improve the modeling, special attention has to be given to match the results obtained from the Hauser-Feshbach modeling to the calculations carried out in the region of isolated resonances. Both should yield reasonable agreement in their respective extrapolations to the unresolved resonance region. Work along these lines is underway.

\section{A. Acknowledgments}

This work was performed under the auspices of the U.S. Department of Energy by Lawrence Livermore National Laboratory under contract DE-AC52-07NA27344. This document has the release number LLNL-TR643852 .
[1] F. Bečvář, Simulation of cascades in complex nuclei with emphasis on assessment of uncertainties of cascaderelated quantities, Nuclear Instruments and Methods in Physics Research Section A: Accelerators, Spectrometers, Detectors and Associated Equipment, 417 (1998), pp. $434-449$.

[2] R. Capote, M. Herman, P. Oblozinský, P. Young, S. Goriely, T. Belgya, A. Ignatyuk, A. Koning, S. Hilaire, V. Plujko, M. Avrigeanu, O. Bersillon, M. Chadwick, T. Fukahori, Z. Ge, Y. Han, S. Kailas, J. Kopecky, V. Maslov, G. Reffo, M. Sin, E. Soukhovitskii, and P. Talou, Ripl - reference input parameter library for calculation of nuclear reactions and nuclear data evaluations, Nuclear Data Sheets, 110 (2009), pp. 3107 3214.

[3] H. Choi, R. Firestone, et al., Radiative capture cross sections of $g d$ isotopes for thermal neutrons. in preparation, 2013.

[4] R. Firestone, K. Abusaleem, and M. Basunia, EGAF: Measurement and analysis of gamma-ray cross sections, Nuclear Data Sheets, submitted (2013).

[5] W. Hauser and H. Feshbach, The inelastic scattering of neutrons, Phys. Rev., 87 (1952), pp. 366-373.

[6] M. Herman, R. Capote, B. Carlson, P. Obložinský, M. Sin, A. Trkov, H. Wienke, and V. Zerkin, EMPIRE: Nuclear reaction model code system for data evaluation, Nuclear Data Sheets, 108 (2007), pp. 2655 - 2715.

[7] A. Hurst, R. Firestone, et al., New measurement of the thermal-capture cross section for the minor isotope ${ }^{180} \mathrm{~W}$, Nuclear Data Sheets, submitted (2013).

[8] — A structural evaluation of the tungsten isotopes via thermal neutron capture. in preparation, 2013.

[9] Hurst, A.M., Firestone, R.B., Sleaford, B.W., Summers, N.C., Revay, Zs., Szentmiklósi, L., Belgya, T., Basunia, M.S., Capote, R., Choi, H., Dashdorj, D., Escher, J., Krticka, M., and Nichols, A., Thermal neutron capture onto the stable tungsten isotopes, EPJ Web of Conferences, 21 (2012), p. 10005.

[10] A. Koning and D. Rochman, Modern nuclear data evaluation with the TALYS code system, Nuclear Data Sheets, 113 (2012), pp. $2841-2934$.

[11] A. J. Koning and J.-P. Delaroche, Local and global nucleon optical models from $1 \mathrm{keV}$ to $200 \mathrm{MeV}$, Nucl. Phys., A713 (2003), p. 231.

[12] S. F. Mughabghab, Atlas of Neutron Resonances, Resonance Parameters and Thermal Cross Sections $Z=1-100$, Elsevier, Amsterdam, 5th ed., 2006.

[13] J. Raynal, Tech. Rep. CEA-N-2772, Commissariat à l'Energie Atomique, Paris, 1994, 1994.

[14] B. Sleaford, R. Firestone, N. Summers, J. Escher, et al., Capture gamma-ray libraries for nuclear applications, Journal of the Korean Physical Society, 59 (2011), p. 1473.

[15] N. Summers, Status of gamma-ray spectra in ENDF (review presentation). 2012.

[16] N. Summers, B. Sleaford, and J. Escher. in preparation.

[17] M. Uhl and B. Strohmaier, Stapre, a computer code for particle induced activation cross sections and related quantities, Tech. Rep. IRK 76/01, rev. 1978, Institut für Radiumforschung und Kernphysik, Vienna, Austria, 1976. 